\title{
Assessment of Fruit Infestation of Abelmoschus esculentus by Shoot and Fruit Borer on Weight Basis
}

\author{
A.R. Lakhamapure ${ }^{1}$, Sh.N.V. Lavhe ${ }^{1 *}$, P.R. Panchabhai ${ }^{2}$, \\ P.S. Barde ${ }^{1}$ and R.O. Deotale ${ }^{1}$ \\ ${ }^{1}$ Entomology Section, College of Agriculture, Nagpur, India \\ ${ }^{2}$ Entomology Section, College of Agriculture, Akola, India
}

*Corresponding author

\section{A B S T R A C T}

\section{Keywords}

Abelmoschus esculentus L., Earias vitella, Shoot infestation, Fruit damage, Weight basis

Article Info

Accepted:

24 August 2018

Available Online:

10 December 2018
To assess the shoot infestation and fruit damage of Abelmoschus esculentus on weight basis the experiment was undertaken during kharif season of 2016-17 in field of Insectory premises of Entomology Section, College of Agriculture, Nagpur. The trial incorporating nine treatments including control which were replicated thrice in Randomized Block Design. During this investigation, on the basis of above parameters revealed that, lowest infestation (shoot and fruit infestation) and highest yield over control was observed in treatment of Spinosad $45 \mathrm{SC} 0.01 \%\left(\mathrm{~T}_{8} ; 6.52 \%\right)$ shoot infestation, $16.13 \%$ fruit damage with $86.79 \mathrm{q} /$ ha yield followed by Emamectin benzoate $5 \mathrm{SG} 0.02 \%\left(\mathrm{~T}_{7}\right)$, Indoxacarb 15.5 EC $0.01 \%\left(\mathrm{~T}_{5}\right)$, Acetamiprid 20 SP $0.2 \mathrm{~g} / \mathrm{lit}\left(\mathrm{T}_{6}\right)$ Bacillus thuringensis $2 \mathrm{ml} / 1\left(\mathrm{~T}_{4}\right)$, Neem oil $2 \%\left(\mathrm{~T}_{1}\right)$, NSE 5\% $\left(\mathrm{T}_{3}\right)$ and Karanj oil 2\% $\left(\mathrm{T}_{2}\right)$.

\section{Introduction}

Okra (Abelmoschus esculentus L.) is one of the most popular vegetable crop grown extensively all over India. However, summer and kharif are the favourable seasons for its cultivation. The area under okra cultivation in India is $4,09,000$ ha with production of $41,93,000$ metric tonnes. Whereas, in Maharashtra State it is cultivated on $26,2,030.3$ ha area with production of 1 , 65,900.4 metric tonnes (Anonymous, 2015). Okra crop attacked by several insect pests like jassids, Amarasca devastans (Dist.); aphids, Aphis gossypii (Glov.); shoot and fruit borer, Earias vitella (Fab.) and Earias insulana
(Biosd.). Among these pests, shoot and fruit borer, Earias vitella (Fabricious). (Noctuidae: Lepidoptera) is the most noxious and destructive pest. Hence, in present study we determined Per cent fruit damage by shoot and fruit borer on 3, 7 and 14 days after $1^{\text {st }}, 2^{\text {nd }}$ and $3^{\text {rd }}$ spray (on weight basis) of each treatment.

\section{Materials and Methods}

The present field experiment was carried out on Okra crop, variety Akola bahar during kharif season 2016-2017 in the Insectory field of Entomology Section, College of Agriculture, Nagpur. The experiment was laid out in a randomized block design with Nine 
treatments consisting Neem oil 2\% $\left(\mathrm{T}_{1}\right)$, Karanj oil 2\% ( $\left.\mathrm{T}_{2}\right)$, NSE 5\% ( $\left.\mathrm{T}_{3}\right)$, Bacillus thuringensis $2 \mathrm{ml} / 1\left(\mathrm{~T}_{4}\right)$, Indoxacarb $15.5 \mathrm{EC}$ $0.01 \%\left(\mathrm{~T}_{5}\right)$, Acetamiprid $20 \mathrm{SP} 0.2 \mathrm{~g} / \mathrm{lit}\left(\mathrm{T}_{6}\right)$, Emamectin benzoate 5 SG $0.02 \% \quad\left(\mathrm{~T}_{7}\right)$, Spinosad 45 SC0.01\%(T8) including (T9) control (water spray) were evaluated after each spray against Natural enemies and observed the effect on them. The plot size was kept $12.6 \mathrm{~m}^{2}$ with a spacing of $60 \times 45 \mathrm{~cm}$ between rows and plants respectively and recommended agronomical practices were followed. In order to know Per cent fruit damage by shoot and fruit borer on 3, 7 and 14 days after $1^{\text {st }}, 2^{\text {nd }}$ and $3^{\text {rd }}$ spray (on weight basis) of each treatment.

The fruits were plucked from 5 selected plants, they were counted and weighed. Similarly, from those total fruits the infested fruits due to fruit borer were separated, counted and weighed. The data on per cent infestation and per cent damage were calculated by adopting the following formulae.

Number of infested shoots Per cent shoot infestation $=$------------ $\times 100$

Total number of shoots

Per cent fruit

$$
\text { Weight of damage fruits }
$$

Damage (Weight basis) $\times 100$

Total weight of fruits

\section{Results and Discussion}

\section{Cumulative per cent shoot infestation by} shoot and fruit borer

Shoot infestation was noticed at 30 DAS and continue upto 65 DAS. The data on the cumulative per cent shoot infestation presented in Table 1 and illustrated in figure 1. From the results, it was revealed that, all the treatments were significantly superior over control in reducing the shoot infestation.
Among these different treatments, Spinosad $45 \mathrm{SC} 0.01 \%\left(\mathrm{~T}_{8}\right)$ recorded minimum $(6.52 \%)$ shoot infestation and was found to be the best followed by Emamectin benzoate 5 SG $0.02 \%$ $\left(\mathrm{T}_{7} ; 7.09 \%\right)$ and Indoxacarb $15.8 \mathrm{EC} 0.01 \%$ ( $\left.\mathrm{T}_{5} ; 7.50 \%\right)$. Devi et al., (2014) reported $5.00 \%$ shoot infestation with the treatment spinosad $45 \%$ SC and emamectin benzoate 5\% SG $5.50 \%$.

The next effective treatment in reducing per cent shoot infestation was of Acetamiprid 20 SP $0.2 \mathrm{~g} / \mathrm{lit}\left(\mathrm{T}_{6} ; 7.92 \%\right)$ and it was followed by Bacillus (B.t.) $\left(1 \times 10^{8} \mathrm{CFU}\right) 2 \mathrm{ml} / \mathrm{lit}\left(\mathrm{T}_{4}\right.$; $8.51 \%$ ). Puranik et al., (2002) reported $11.07 \%$ shoot infestation of $L$. orbonalis with the treatment halt WP $(B t)$.

The next promising treatment in reducing per cent shoot infestation was of Neem oil $2 \%\left(\mathrm{~T}_{1}\right.$; $8.83 \%)$ followed by NSE $5 \%\left(\mathrm{~T}_{3} ; 8.94 \%\right)$. Both these treatments were statistically at par with each other. Our present findings regarding efficacy of neem oil 2\% $\left(\mathrm{T}_{5}\right)$ confirmed the findings of Panzade (2006), who recorded $7.95 \%$, shoot infestation with the treatment of neem oil $1 \%$.

The Karanj oil 2\% $\left(\mathrm{T}_{2} ; 9.06 \%\right)$ showed least effectiveness in reducing per cent shoot infestation but found to be superior over control $\left(\mathrm{T}_{9} ; 10.29 \%\right)$.

The data pertaining to per cent fruit damage (Table 2) indicated that, all the treatments were significantly superior over control, 3 DAT. The treatment of Spinosad 45 SC $0.01 \%$ $\left(\mathrm{T}_{8}\right)$ recorded lowest $(18.30 \%)$ fruit damage followed by Emamectin benzoate 5 SG $0.02 \%$ $\left(\mathrm{T}_{7} ; 19.37 \%\right)$, Indoxacarb $15.8 \mathrm{EC} 0.01 \%\left(\mathrm{~T}_{5}\right.$; $21.10 \%)$, Acetamiprid 20 SP $0.2 \mathrm{~g} / \mathrm{lit}\left(\mathrm{T}_{6}\right.$; 21.80) and Bacillus thuringensis $2 \mathrm{ml} / \mathrm{l}\left(\mathrm{T}_{4}\right.$; $21.95 \%$ ) and all these treatments were statistically at par with the treatment of Spinosad $45 \mathrm{SC} 0.01 \%\left(\mathrm{~T}_{8}\right)$. Priya and Mishra (2007) recorded $43.95 \%$ fruit infestation on 
weight basis with the treatment of spinosad 45\% SC. Anil and Sharma (2010) recorded lowest fruit infestation with emamectin benzoate 5 SG $0.002 \%$. Bansode et al., (2014) reported that minimum fruit damage was observed with indoxacarb @ $75 \mathrm{~g}$ a.i./ha.

The next effective treatment was of Neem oil $2 \%\left(\mathrm{~T}_{1} ; 23.88 \%\right)$ and NSE $5 \%\left(\mathrm{~T}_{3} ; 24.01 \%\right)$ and both these treatments were statistically at par with each other.

The treatment of Karanj oil 2\% ( $\left.\mathrm{T}_{2} ; 25.03 \%\right)$ was found to be least effective but superior over control $\left(\mathrm{T}_{9}\right)$ in which $29.11 \%$ fruit damage was recorded.

The results on 7 days after first spray indicated that, all treatments were significantly superior over control in lowering fruit damage. The treatment of Spinosad 45 SC $0.01 \% \quad\left(\mathrm{~T}_{8}\right)$ recorded $19.50 \%$ fruit damage followed by Emamectin benzoate 5 SG $0.02 \% \quad\left(\mathrm{~T}_{7}\right.$; $20.70 \%$ ), Indoxacarb 15.8 EC $0.01 \% \quad\left(\mathrm{~T}_{5}\right.$; 21.85\%), Acetamiprid 20 SP $0.2 \mathrm{~g} / \mathrm{lit}\left(\mathrm{T}_{6}\right.$; 22.75) and Bacillus thuringensis $2 \mathrm{ml} / \mathrm{l}\left(\mathrm{T}_{4}\right.$; $22.75 \%)$.

The next effective treatments were of Neem oil $2 \%\left(\mathrm{~T}_{1} ; 24.13 \%\right)$ and $\mathrm{NSE} 5 \% \quad\left(\mathrm{~T}_{3}\right.$; $25.15 \%)$ and both these treatments were at par with each other.

The treatment of Karanj oil 2\% $\left(\mathrm{T}_{2} ; 26.85 \%\right)$ was least effective in reducing fruit damage but found to be superior over control $\left(\mathrm{T}_{9}\right.$; $31.15 \%)$.

Similar type of results were obtained 14 days after first spray in which the treatment of Spinosad $45 \mathrm{SC} 0.01 \%\left(\mathrm{~T}_{8}\right)$ recorded the lowest $(20.80 \%)$ infestation followed by promising treatments of Emamectin benzoate 5 SG $0.02 \%\left(\mathrm{~T}_{7} ; 21.50 \%\right)$, Indoxacarb 15.8 EC $0.01 \%\left(\mathrm{~T}_{5} ; 22.50 \%\right)$, Acetamiprid $20 \mathrm{SP}$ 0.2 g/lit $\left(\mathrm{T}_{6} ; 23.15 \%\right)$, Bacillus thuringensis 2 $\mathrm{ml} / \mathrm{l}\left(\mathrm{T}_{4} ; 23.15 \%\right)$ and these treatments were statistically at par with the treatment of Spinosad of $45 \mathrm{SC} 0.01 \%\left(\mathrm{~T}_{8}\right)$.

In botanicals promising treatments were of Neem oil $2 \%\left(\mathrm{~T}_{1} ; 25.66 \%\right)$, NSE $5 \%\left(\mathrm{~T}_{3}\right.$; $27.60 \%$ ) and these treatment were statistically at par with each other.

The treatment regarding Karanj oil 2\% $\left(\mathrm{T}_{2}\right)$ showed higher infestation $29.00 \%$ but found to be superior over control $\left(\mathrm{T}_{9}\right)$, where $32.30 \%$ infestation was recorded. As the results on this parameter are also studied after second and third spray, the results are not discussed here.

They are discussed further in results obtained after second and third spray.

\section{Per cent fruit damage by shoot and fruit borer, 3, 7 and 14 days after second spray (on weight basis)}

The data presented in Table 2 revealed that, the spraying of Spinosad $45 \mathrm{SC} 0.01 \%\left(\mathrm{~T}_{8}\right)$ offered the best protection against E.vittella in reducing per cent fruit damage on weight basis $(16.20 \%)$ followed by Emamectin benzoate 5 SG $0.02 \%\left(\mathrm{~T}_{7} ; 19.18 \%\right)$, Indoxacarb $15.8 \mathrm{EC}$ $0.01 \%\left(\mathrm{~T}_{5} ; 21.01 \%\right)$, Acetamiprid 20 SP 0.2 $\mathrm{g} / \mathrm{lit}\left(\mathrm{T}_{6} ; 21.42 \%\right)$ and Bacillus thuringensis 2 $\mathrm{ml} / \mathrm{l}\left(\mathrm{T}_{4} ; 22.02 \%\right)$. Priya and Mishra (2007) recorded $43.95 \%$ fruit infestation on weight basis with the treatment spinosad $45 \%$ SC. Anil and Sharma (2010) recorded lowest fruit infestation with emamectin benzoate $5 \mathrm{SG}$ 0.02\%. Mallapur et al., (2012) found indoxacarb 14.5SC @ 500 ml/ha effective in controlling fruit infestation.

The next effective treatments were of Neem oil $2 \% \quad\left(\mathrm{~T}_{1} ; 22.80 \%\right)$ and NSE $5 \% \quad\left(\mathrm{~T}_{3}\right.$; $23.82 \%$ ) and both these treatments were statistically at par with each other. The treatment of Karanj oil 2\% (T2; 24.77\%) found to be least effective. 
Fig.1 Cumulative per cent shoot infestation by shoot and fruit borer

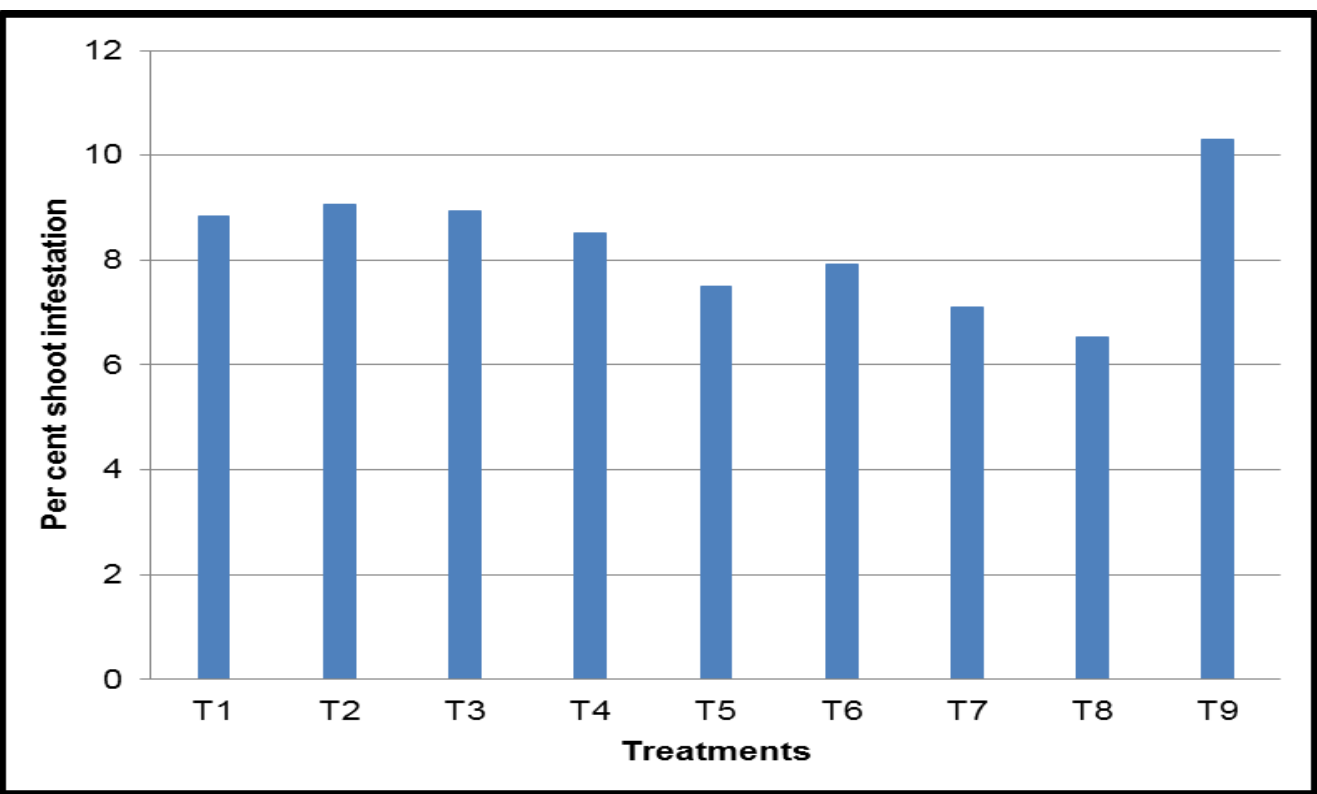

$\mathrm{T}_{1}=$ Neem oil $-2 \% ; \mathrm{T}_{2}=$ Karanj oil $-2 \% ; \mathrm{T}_{3}=\mathrm{NSE}-5 \%$

$\mathrm{T}_{4}=$ Bacillus thuringiensis $-2 \mathrm{ml} / \mathrm{lit} ; \mathrm{T}_{5}=$ Indoxacarb $15.8 \mathrm{EC}-0.01 \%$

$\mathrm{T}_{6}=$ Acetamiprid $20 \mathrm{SP}-0.2 \mathrm{~g} / \mathrm{lit} ; \mathrm{T}_{7}=$ Emamectin Benzoate $5 \mathrm{SG}-0.02 \% ; \mathrm{T}_{8}=$ Spinosad $45 \mathrm{SC}-0.01 \% ; \mathrm{T}_{9}=$ Control (water spray)

Per cent fruit damage by shoot and fruit borer, 3, 7 and 14 days after first spray (on weight basis)

Fig.2 Cumulative per cent fruit damage by shoot and fruit borer (on weight basis)

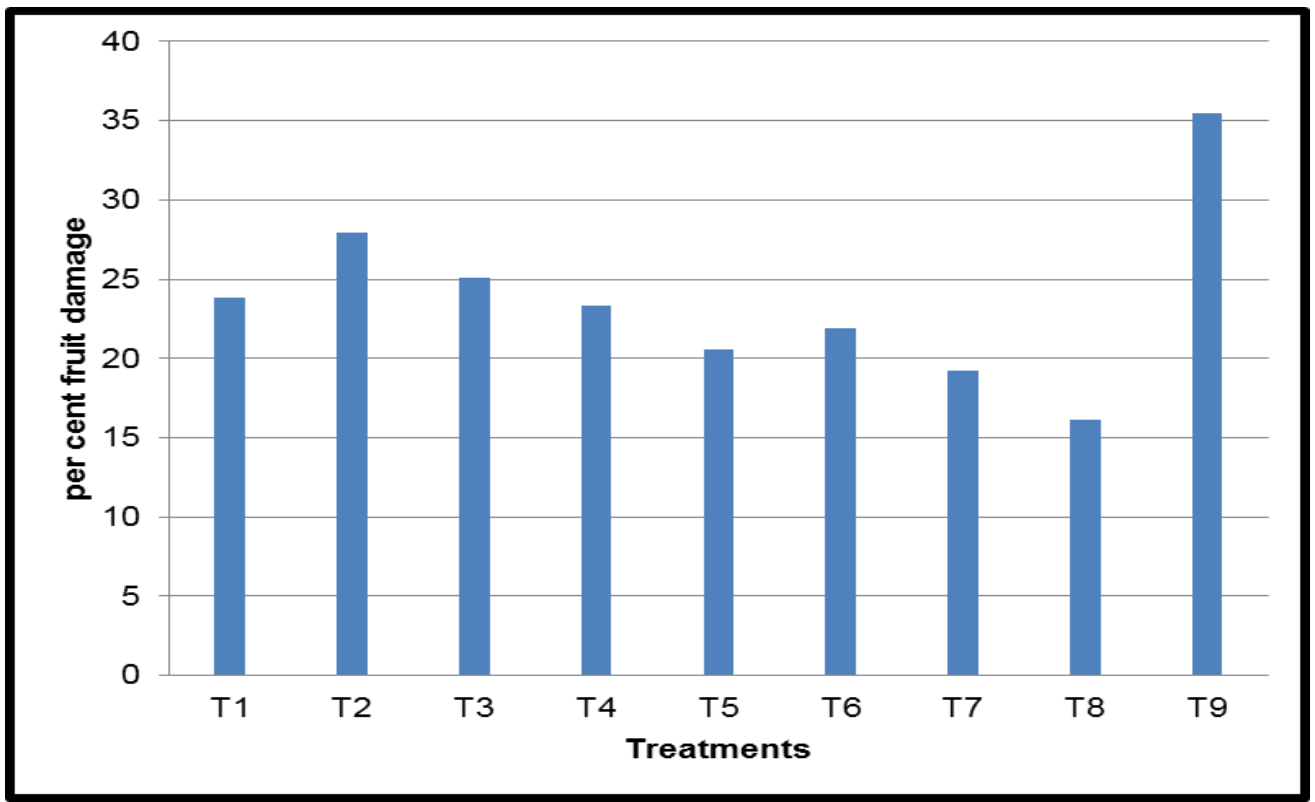

$\mathrm{T}_{1}=$ Neem oil $-2 \% ; \mathrm{T}_{2}=$ Karanj oil $-2 \% ; \mathrm{T}_{3}=\mathrm{NSE}-5 \%$

$\mathrm{T}_{4}=$ Bacillus thuringiensis $-2 \mathrm{ml} / \mathrm{lit} ; \mathrm{T}_{5}=$ Indoxacarb $15.8 \mathrm{EC}-0.01 \%$

$\mathrm{T}_{6}=$ Acetamiprid $20 \mathrm{SP}-0.2 \mathrm{~g} / \mathrm{lit} ; \mathrm{T}_{7}=$ Emamectin Benzoate $5 \mathrm{SG}-0.02 \% ; \mathrm{T}_{8}=$ Spinosad $45 \mathrm{SC}-0.01 \% ; \mathrm{T}_{9}=$ Control (water spray) 


\section{Int.J.Curr.Microbiol.App.Sci (2018) 7(12): 3503-3512}

Table.1 Cumulative per cent shoot infestation by shoot and fruit borer

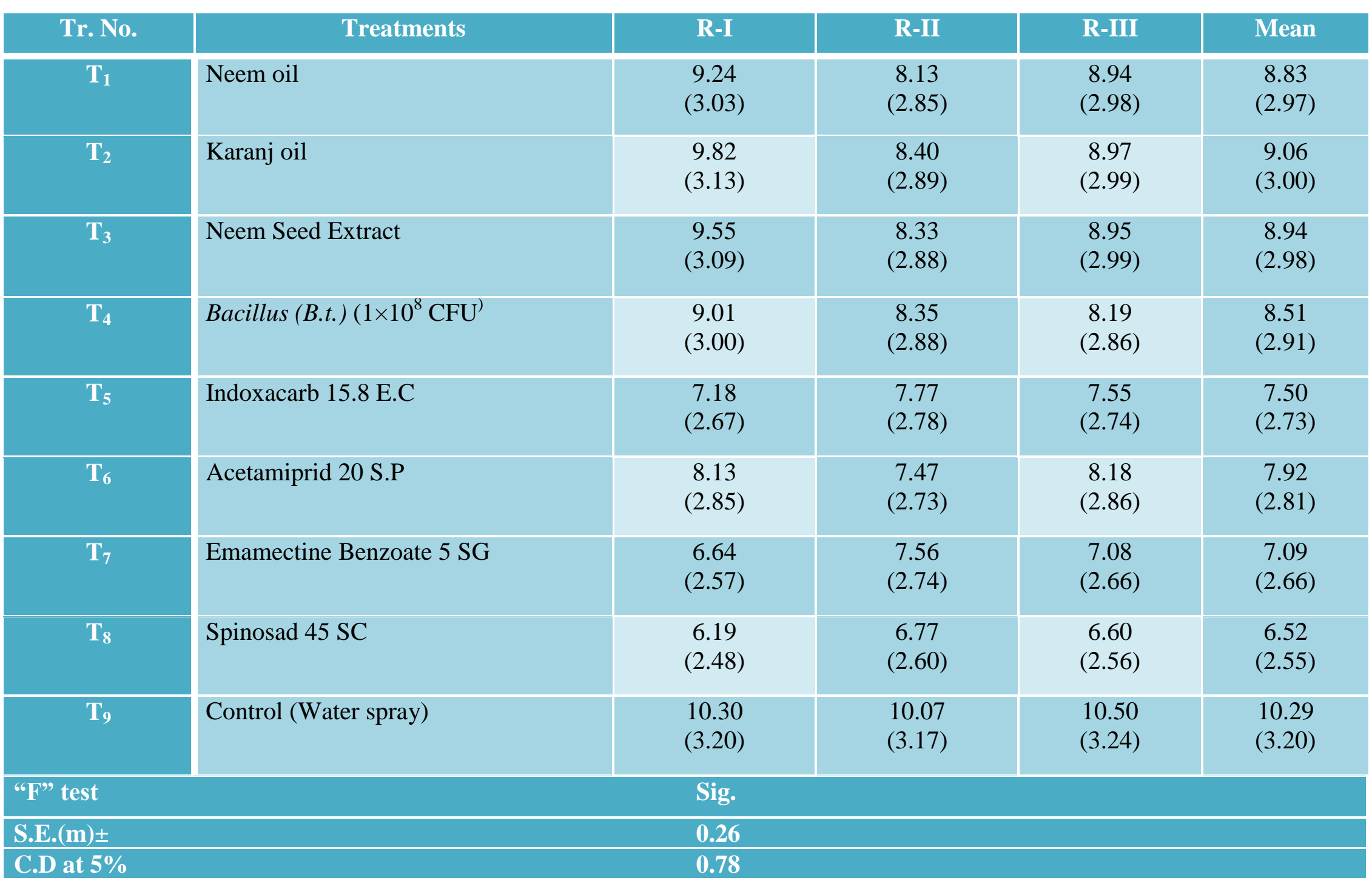

(Figures in parenthesis are square root transformed values.) 
Table.2 Mean per cent fruit infestation by shoot and fruit borer (on weight basis)

\begin{tabular}{|c|c|c|c|c|c|c|c|c|c|}
\hline \multirow[t]{2}{*}{ Treatments } & \multicolumn{3}{|c|}{$1^{\text {st }}$ spray } & \multicolumn{3}{|c|}{$2^{\text {nd }}$ spray } & \multicolumn{3}{|c|}{$3^{\text {rd }}$ spray } \\
\hline & 3DAT & 7DAT & 14DAT & 3DAT & 7DAT & 14DAT & 3DAT & 7DAT & 14DAT \\
\hline Neem oil & $\begin{array}{c}23.88 \\
(29.25)\end{array}$ & $\begin{array}{c}24.13 \\
(29.42)\end{array}$ & $\begin{array}{c}25.66 \\
(30.43)\end{array}$ & $\begin{array}{c}22.80 \\
(28.52)\end{array}$ & $\begin{array}{c}23.50 \\
(28.99)\end{array}$ & $\begin{array}{c}25.35 \\
(30.23)\end{array}$ & $\begin{array}{c}23.09 \\
(28.71)\end{array}$ & $\begin{array}{c}23.45 \\
(28.96)\end{array}$ & $\begin{array}{c}24.24 \\
(29.49)\end{array}$ \\
\hline Karanj oil & $\begin{array}{c}25.03 \\
(30.01)\end{array}$ & $\begin{array}{c}26.85 \\
(31.20)\end{array}$ & $\begin{array}{c}29.00 \\
(32.58)\end{array}$ & $\begin{array}{c}24.77 \\
(29.84)\end{array}$ & $\begin{array}{c}26.85 \\
(31.20)\end{array}$ & $\begin{array}{c}28.09 \\
(32.00)\end{array}$ & $\begin{array}{c}24.80 \\
(29.86)\end{array}$ & $\begin{array}{c}25.80 \\
(30.52)\end{array}$ & $\begin{array}{c}26.80 \\
(31.17)\end{array}$ \\
\hline Neem Seed Extract & $\begin{array}{c}24.01 \\
(29.34)\end{array}$ & $\begin{array}{c}25.15 \\
(30.09)\end{array}$ & $\begin{array}{c}27.60 \\
(31.69)\end{array}$ & $\begin{array}{c}23.82 \\
(29.21)\end{array}$ & $\begin{array}{c}24.60 \\
(29.73)\end{array}$ & $\begin{array}{c}25.90 \\
(30.59)\end{array}$ & $\begin{array}{c}23.50 \\
(28.99)\end{array}$ & $\begin{array}{c}23.80 \\
(29.19)\end{array}$ & $\begin{array}{c}24.95 \\
(29.96)\end{array}$ \\
\hline Bacillus (B.t.) $\left(1 \times 10^{8} \mathrm{CFU}^{)}\right.$ & $\begin{array}{c}21.95 \\
(27.93)\end{array}$ & $\begin{array}{c}22.75 \\
(28.48)\end{array}$ & $\begin{array}{c}23.15 \\
(28.76)\end{array}$ & $\begin{array}{c}22.02 \\
(27.98)\end{array}$ & $\begin{array}{c}22.98 \\
(28.64)\end{array}$ & $\begin{array}{c}24.07 \\
(29.38)\end{array}$ & $\begin{array}{c}22.04 \\
(27.99)\end{array}$ & $\begin{array}{c}22.80 \\
(28.52)\end{array}$ & $\begin{array}{c}23.80 \\
(29.19)\end{array}$ \\
\hline Indoxacarb 15.8 E.C & $\begin{array}{c}21.10 \\
(27.34)\end{array}$ & $\begin{array}{c}21.85 \\
(27.86)\end{array}$ & $\begin{array}{c}22.50 \\
(28.31)\end{array}$ & $\begin{array}{c}21.01 \\
(27.28)\end{array}$ & $\begin{array}{c}21.55 \\
(27.65)\end{array}$ & $\begin{array}{c}21.70 \\
(27.76)\end{array}$ & $\begin{array}{c}21.01 \\
(27.28)\end{array}$ & $\begin{array}{c}21.10 \\
(27.34)\end{array}$ & $\begin{array}{c}22.22 \\
(28.12)\end{array}$ \\
\hline Acetamiprid 20 S.P & $\begin{array}{c}21.80 \\
(27.83)\end{array}$ & $\begin{array}{c}22.75 \\
(28.48)\end{array}$ & $\begin{array}{c}23.15 \\
(28.76)\end{array}$ & $\begin{array}{c}21.42 \\
(27.56)\end{array}$ & $\begin{array}{c}22.25 \\
(28.14)\end{array}$ & $\begin{array}{c}23.24 \\
(28.82)\end{array}$ & $\begin{array}{c}21.80 \\
(27.83)\end{array}$ & $\begin{array}{c}22.23 \\
(28.13)\end{array}$ & $\begin{array}{c}23.01 \\
(28.66) \\
\end{array}$ \\
\hline Emamectine Benzoate 5 SG & $\begin{array}{c}19.37 \\
(26.11)\end{array}$ & $\begin{array}{c}20.70 \\
(27.06)\end{array}$ & $\begin{array}{c}21.50 \\
(27.62)\end{array}$ & $\begin{array}{c}19.18 \\
(25.97)\end{array}$ & $\begin{array}{c}20.35 \\
(26.81)\end{array}$ & $\begin{array}{c}21.18 \\
(27.40)\end{array}$ & $\begin{array}{c}18.17 \\
(25.23)\end{array}$ & $\begin{array}{c}19.35 \\
(26.09)\end{array}$ & $\begin{array}{c}20.79 \\
(27.12)\end{array}$ \\
\hline Spinosad 45 SC & $\begin{array}{c}18.30 \\
(25.32)\end{array}$ & $\begin{array}{c}19.50 \\
(26.20)\end{array}$ & $\begin{array}{c}20.80 \\
(27.13)\end{array}$ & $\begin{array}{c}16.20 \\
(23.73)\end{array}$ & $\begin{array}{c}17.80 \\
(24.95)\end{array}$ & $\begin{array}{c}20.30 \\
(26.77)\end{array}$ & $\begin{array}{c}9.08 \\
(17.53)\end{array}$ & $\begin{array}{c}10.75 \\
(19.13)\end{array}$ & $\begin{array}{c}12.50 \\
(20.70)\end{array}$ \\
\hline Control (Water spray) & $\begin{array}{c}29.11 \\
(32.65)\end{array}$ & $\begin{array}{c}31.15 \\
(33.92)\end{array}$ & $\begin{array}{c}32.30 \\
(34.63)\end{array}$ & $\begin{array}{c}35.39 \\
(36.50)\end{array}$ & $\begin{array}{c}36.65 \\
(37.25)\end{array}$ & $\begin{array}{c}37.80 \\
(37.93)\end{array}$ & $\begin{array}{c}38.34 \\
(38.25)\end{array}$ & $\begin{array}{c}38.65 \\
(38.43)\end{array}$ & $\begin{array}{c}40.08 \\
(39.27) \\
\end{array}$ \\
\hline F Test & Sig & Sig & Sig & Sig & Sig & Sig & Sig & Sig & Sig \\
\hline $\operatorname{SEm}( \pm)$ & 1.46 & 1.53 & 1.62 & 1.46 & 1.53 & 1.61 & 1.45 & 1.49 & 1.56 \\
\hline CD@ $@ 5 \%$ & 4.38 & 4.59 & 4.86 & 4.37 & 4.60 & 4.83 & 4.35 & 4.47 & 4.68 \\
\hline
\end{tabular}

(Figures in parenthesis are arcs in transformed values.) 
Table.3 Cumulative per cent fruit infestation by shoot and fruit borer (On weight basis)

\begin{tabular}{|c|c|c|c|c|c|}
\hline Tr. No. & Treatments & $\mathbf{R}-\mathbf{I}$ & R-II & R-III & Mean \\
\hline $\mathbf{T}_{1}$ & Neem oil & $\begin{array}{c}23.70 \\
(29.13)\end{array}$ & $\begin{array}{c}21.79 \\
(27.82)\end{array}$ & $\begin{array}{c}26.12 \\
(30.73)\end{array}$ & $\begin{array}{c}23.87 \\
(29.24)\end{array}$ \\
\hline $\mathbf{T}_{2}$ & Karanj oil & $\begin{array}{c}25.15 \\
(30.09)\end{array}$ & $\begin{array}{c}29.50 \\
(32.89)\end{array}$ & $\begin{array}{c}29.07 \\
(32.62)\end{array}$ & $\begin{array}{c}27.90 \\
(31.88)\end{array}$ \\
\hline $\mathbf{T}_{3}$ & Neem Seed Extract & $\begin{array}{c}23.78 \\
(29.18)\end{array}$ & $\begin{array}{c}25.20 \\
(30.13)\end{array}$ & $\begin{array}{c}26.30 \\
(30.85)\end{array}$ & $\begin{array}{c}25.09 \\
(30.05)\end{array}$ \\
\hline $\mathbf{T}_{4}$ & Bacillus (B.t.) $\left(1 \times 10^{8} \mathrm{CFU}^{)}\right.$ & $\begin{array}{c}22.28 \\
(28.16)\end{array}$ & $\begin{array}{c}21.58 \\
(27.68)\end{array}$ & $\begin{array}{c}26.01 \\
(30.66)\end{array}$ & $\begin{array}{c}23.29 \\
(28.85)\end{array}$ \\
\hline $\mathbf{T}_{5}$ & Indoxacarb 15.8 E.C & $\begin{array}{c}20.94 \\
(27.23)\end{array}$ & $\begin{array}{c}20.58 \\
(26.97)\end{array}$ & $\begin{array}{c}20.19 \\
(26.70)\end{array}$ & $\begin{array}{c}20.57 \\
(26.97)\end{array}$ \\
\hline $\mathbf{T}_{6}$ & Acetamiprid 20 S.P & $\begin{array}{c}21.46 \\
(27.59)\end{array}$ & $\begin{array}{c}21.57 \\
(27.67)\end{array}$ & $\begin{array}{c}22.70 \\
(28.45)\end{array}$ & $\begin{array}{c}21.91 \\
(27.90)\end{array}$ \\
\hline $\mathbf{T}_{7}$ & Emamectine Benzoate $5 \mathrm{SG}$ & $\begin{array}{c}17.54 \\
(24.75)\end{array}$ & $\begin{array}{c}20.52 \\
(26.93)\end{array}$ & $\begin{array}{c}19.72 \\
(26.36)\end{array}$ & $\begin{array}{c}19.26 \\
(26.03)\end{array}$ \\
\hline $\mathbf{T}_{8}$ & Spinosad $45 \mathrm{SC}$ & $\begin{array}{c}17.02 \\
(24.36)\end{array}$ & $\begin{array}{c}16.37 \\
(23.86)\end{array}$ & $\begin{array}{c}15.00 \\
(22.78)\end{array}$ & $\begin{array}{c}16.13 \\
(23.67)\end{array}$ \\
\hline T9 & Control (Water spray) & $\begin{array}{c}34.73 \\
(36.10)\end{array}$ & $\begin{array}{c}38.15 \\
(38.14)\end{array}$ & $\begin{array}{c}33.59 \\
(35.42)\end{array}$ & $\begin{array}{c}35.49 \\
(36.56)\end{array}$ \\
\hline "F" test & \multicolumn{5}{|c|}{ Sig. } \\
\hline S.E. $(m) \pm$ & \multicolumn{5}{|c|}{0.98} \\
\hline C.D at $5 \%$ & \multicolumn{5}{|c|}{2.93} \\
\hline
\end{tabular}

(Figures in parenthesis are arcs in transformed values.) 
Similar results were obtained 7 days after second spray. The results indicated that, spraying of Spinosad 45 SC $0.01 \% \quad\left(\mathrm{~T}_{8}\right)$ recorded lowest fruit damage $(17.80 \%)$ followed by Emamectin benzoate 5 SG $0.02 \%$ $\left(\mathrm{T}_{7} ; 20.35 \%\right)$, Indoxacarb 15.8 EC $0.01 \%\left(\mathrm{~T}_{5}\right.$; $21.55 \%)$, Acetamiprid 20 SP 0.2 g/lit $\left(\mathrm{T}_{6}\right.$; $22.25 \%)$ and Bacillus thuringensis $2 \mathrm{ml} / \mathrm{l}\left(\mathrm{T}_{4}\right.$; $22.98 \%)$.

The next promising treatments were of Neem oil 2\% $\left(\mathrm{T}_{1} ; 23.50 \%\right)$ and NSE 5\% $\left(\mathrm{T}_{3} ; 24.60 \%\right)$ and both these treatments were statistically comparable with each other. Our present results on efficacy of NSE 5\% $\left(\mathrm{T}_{4}\right)$ corroborate with the findings of Ambekar et al., (2000). They reported $27.92 \%$ fruit damage with the treatment NSE 5\%. Malik and Lal (1989) reported the effectiveness of neem oil and our present findings are in agreement with their results. Desai and Kapadia (2006) reported $61.22 \%$ larval mortality under laboratory condition with the treatment Btk@1 kg/ha and our present results could be comparable with their reports.

The treatment of Karanj oil 2\% $\left(\mathrm{T}_{2} ; 26.85 \%\right)$ was found to be least effective but superior over control $\left(\mathrm{T}_{8} ; 36.65 \%\right)$.

Similar type of results were obtained 14 days after second spray in which the treatment of Spinosad 45 SC $0.01 \%\left(\mathrm{~T}_{8}\right)$ recorded the lowest (20.30\%) infestation followed by promising Emamectin benzoate 5 SG $0.02 \%\left(\mathrm{~T}_{7} ; 21.18 \%\right)$, Indoxacarb 15.8 EC $0.01 \% \quad\left(\mathrm{~T}_{5} ; 21.70 \%\right)$, Acetamiprid 20 SP $0.2 \mathrm{~g} / \mathrm{lit}\left(\mathrm{T}_{6} ; 23.24 \%\right)$, Bacillus thuringensis $2 \mathrm{ml} / \mathrm{l}\left(\mathrm{T}_{4} ; 24.07 \%\right)$ and these treatment were statistically at par with the treatment of Spinosad of $45 \mathrm{SC} 0.01 \%\left(\mathrm{~T}_{8}\right)$.

In botanicals promising treatments were of Neem oil $2 \%\left(\mathrm{~T}_{1} ; 25.35 \%\right)$, NSE $5 \% \quad\left(\mathrm{~T}_{3}\right.$; $25.90 \%)$ and these treatment were statistically at par with each other. The treatment regarding Karanj oil 2\% ( $\left.\mathrm{T}_{2}\right)$ showed higher infestation $28.09 \%$ but found to be superior over control $\left(\mathrm{T}_{9}\right)$, where $38.34 \%$ infestation was recorded.
Per cent fruit damage by shoot and fruit borer, 3, 7 and 14 days after third spray (on weight basis)

The data pertaining to per cent fruit damage (Table 2) indicated that, the Spinosad 45 SC $0.01 \% \quad\left(\mathrm{~T}_{8}\right)$ recorded lowest $9.08 \%$ fruit damage. Next promising treatments were of Emamectin benzoate $5 \mathrm{SG} 0.02 \%\left(\mathrm{~T}_{7} ; 18.17 \%\right)$, Indoxacarb 15.8 EC $0.01 \% \quad\left(\mathrm{~T}_{5} ; 21.01 \%\right)$, Acetamiprid 20 SP $0.2 \mathrm{~g} / \mathrm{lit}\left(\mathrm{T}_{6} ; 21.80 \%\right)$ and Bacillus thuringensis $2 \mathrm{ml} / 1\left(\mathrm{~T}_{4} ; 22.04 \%\right)$ and all these treatments were at par with each other. Priya and Mishra (2007) recorded 43.95\% fruit infestation on weight basis with the treatment of spinosad $45 \%$ SC.

Anil and Sharma (2010) recorded lowest fruit infestation with emamectin benzoate $5 \mathrm{SG}$ $0.002 \%$. Mallapur et al., (2012) reported that minimum fruit damage was observed with indoxacarb 14.5SC @ 500 ml/ha.

The next promising treatments were of Neem oil $2 \%\left(\mathrm{~T}_{1} ; 23.09 \%\right)$, NSE $5 \%\left(\mathrm{~T}_{3} ; 23.50 \%\right)$ and Karanj oil $2 \%\left(\mathrm{~T}_{2} ; 24.80 \%\right)$ and these treatments were statistically comparable with each other.

Rasoaiah (2001) reported NSE 5\% as highly promising against E.vittella. Malik and Lal (1989), Pathak and Krishna (1986) reported the effect of neem oil against E.vittella and our present findings agree with their reports. Kharbade et al., (1999) reported the efficacy of Bt. against E.vittella.

The treatment of Karanj oil 2\% $\left(\mathrm{T}_{2}\right)$ was found to be least effective and recorded $24.80 \%$ fruit infestation but found to be superior over control $\left(\mathrm{T}_{9} ; 37.80 \%\right)$.

Similar results obtained 7 days after third spray, the data revealed that, the treatment of Spinosad $45 \mathrm{SC} 0.01 \%\left(\mathrm{~T}_{8}\right)$ recorded lowest fruit damage $(10.75 \%)$ followed by Emamectin benzoate 5 SG $0.02 \%\left(\mathrm{~T}_{7} ; 19.35 \%\right)$, Indoxacarb $15.8 \mathrm{EC}$ $0.01 \%\left(\mathrm{~T}_{5} ; 21.10 \%\right)$, Acetamiprid 20 SP 0.2 $\mathrm{g} / \mathrm{lit}\left(\mathrm{T}_{6} ; 22.23 \%\right)$ and Bacillus thuringensis 2 
$\mathrm{ml} / \mathrm{l}\left(\mathrm{T}_{4} ; 22.80 \%\right)$ which were statistically at par with each other.

The next promising treatments were of Neem oil $2 \%\left(\mathrm{~T}_{1} ; 23.45 \%\right)$, NSE $5 \%\left(\mathrm{~T}_{3} ; 23.80 \%\right)$ and Karanj oil $2 \%\left(\mathrm{~T}_{2} ; 25.80 \%\right)$ and all these treatments were statistically comparable with each other. Rosaiah (2001) and Gajmer et al., (2003) reported the efficacy of NSE against $E$. vittella. Kharbade et al., (1999) and Tomar (1998) reported the effectiveness of $B t$ So, our present findings agree with their reports.

The treatment of Karanj oil 2\% $\left(\mathrm{T}_{2} ; 25.80 \%\right)$ was comparably least effective against shoot and fruit borer. Reddy and Srinivasa reported the effectiveness of pungam oil $2 \%$ for the management of brinjal shoot and fruit borer $L$. orbonalis.

Similar types of results were obtained 14 days after third spray in which the treatment of Spinosad 45 SC $0.01 \%\left(\mathrm{~T}_{8}\right)$ recorded the lowest $(12.50 \%)$ infestation. Next promising treatment were of Emamectin benzoate 5 SG $0.02 \%\left(\mathrm{~T}_{7}\right.$; $20.79 \%)$, Indoxacarb 15.8 EC $0.01 \% \quad\left(\mathrm{~T}_{5}\right.$; 22.22\%), Acetamiprid 20 SP $0.2 \mathrm{~g} / \mathrm{lit} \quad\left(\mathrm{T}_{6}\right.$; $23.01 \%)$, Bacillus thuringensis $2 \mathrm{ml} / \mathrm{l} \quad\left(\mathrm{T}_{4}\right.$; $23.80 \%$ ) and these treatments were statistically at par with the treatment of Emamectin benzoate $5 \mathrm{SG} 0.02 \%\left(\mathrm{~T}_{7}\right)$.

In botanicals promising treatments were of Neem oil $2 \%\left(\mathrm{~T}_{1} ; 24.24 \%\right)$, NSE $5 \%\left(\mathrm{~T}_{3}\right.$; $24.95 \%$ ) and these treatment were statistically at par with each other. The treatment regarding Karanj oil 2\% $\left(\mathrm{T}_{2}\right)$ showed higher infestation $26.80 \%$ but found to be superior over control $\left(\mathrm{T}_{9}\right)$, where $40.08 \%$ infestation was recorded.

\section{Cumulative per cent fruit damage by shoot and fruit borer (On weight basis)}

The data on cumulative per cent fruit damage of shoot and fruit borer computed from all observations of various treatments is presented in Table 3 and illustrated in figure 2. The results revealed that, all the treatments were significantly superior over control. The most effective treatment was of Spinosad $45 \mathrm{SC}$ $0.01 \%\left(\mathrm{~T}_{8}\right)$ which consistently maintained its efficacy in recording minimum fruit damage throughout the period of experimentation with average of $16.13 \%$ fruit infestation followed by the treatments of Emamectin benzoate $5 \mathrm{SG}$ $0.02 \%\left(\mathrm{~T}_{7} ; 20.06 \%\right)$, Indoxacarb $15.8 \mathrm{EC} 0.01 \%$ $\left(\mathrm{T}_{5} ; 21.56 \%\right)$, Acetamiprid $20 \mathrm{SP} 0.2 \mathrm{~g} / \mathrm{lit}\left(\mathrm{T}_{6}\right.$; $22.40 \%)$ and Bacillus thuringensis $2 \mathrm{ml} / \mathrm{l}\left(\mathrm{T}_{4}\right.$; $22.84 \%)$.

The next effective treatments were of Neem oil $2 \%\left(\mathrm{~T}_{1} ; 24.01 \%\right)$, NSE $5 \%\left(\mathrm{~T}_{3} ; 24.81 \%\right)$ and both these treatments were statistically comparable with each other. Sarode and Gabhane (1994) reported $29.49 \%$ fruit damage with the treatment NSE 5\% $\left(\mathrm{T}_{3}\right)$ and our present results agree with their findings. The results on efficacy of neem oil $2 \%\left(\mathrm{~T}_{1}\right)$ confirmed the reports of Malik and Lal (1989). Panzade (2006) recorded $25.90 \%$ fruit damage with treatment Neem oil 2\%. The present results on efficacy of Bacillus thuringensis 2ml/1 $\left(\mathrm{T}_{4}\right)$ agree with the reports of Kharbade et al., (1999). They reported $13.21 \%$ fruit damage 4 DAT with Dipel 8L.

The treatment of Karanj oil 2\% $\left(\mathrm{T}_{2} ; 26.44 \%\right)$ was found to be least effective and recorded higher fruit damage. Reddy and Srinivasa reported the effectiveness of pungam oil $2 \%$ for the management of brinjal shoot and fruit borer L. orbonalis.

\section{References}

Ambekar, J. S., A. S. Pawar, and M. V. Sakhare, 2001. Bio-efficacy of certain neem products against okra fruit borer, J. Maharastra Agri. Univ., 25 (1): 42-43.

Anil and P. C. Sharma, 2010. Bioefficacy of insecticides against Leucinodes orbonalis on brinjal, Journal of environmental biology. 31, 399-402.

Anonymous, 2015. All-India Area, Production and Yield of okra. Directorate of Economics and Statistics, Department of Agriculture and Cooperation. http://www.mahaagri.gov.in. 
Anonymus, 2015. Indian horticulture database. National horticulture board, New Delhi at www.nhb.gov.in

Bansode, A. G., C. S. Patil and S. S. Jadhav, 2015. Efficacy of insecticides against shoot and fruit borer, Earias vittella $\mathrm{F}$. infesting okra Pest Management in Horticultural Ecosystems, 21(1): 106-109.

Desai, V.S. and M.N. Kapadia, 2006. Efficacy of Bacillus thuringiensis var. Kurstaki (Halt) alone and in combination with insecticides under laboratory conditions. Pestology. 30(12): 15-18.

Gajmer, T., Ram Singh, R.K. Saini and S.B. Kalidas. 2003. Growth and development inhibitory effect of Azadirachtin indica and Melia azadarach on E. vitella. J. Medicinal and Aromatic Plant Sci., 25(1): 108-112.

Kharbade, S. B., A.G. Chandele and C.S. Chauudhary, 1999. Comparative efficiacy of Dipel 8L, Delfin WG and halt rock $01 \mathrm{WP}$ against Earias vitella. Pestology. 23(8): 51-52.

Malik, S.N. and I.B. Lal. 1989. Efficacy of neem oil cake and fertilizer mixture against okra fruit borer. Pestology. 13(11): 6-7.

Mallapur, C. P., M. Chouraddi, P. Nayaka, D. N. Dhanalakshmi and R. A. Balikai, 2012. Evaluation of insecticides against insect pest complex of okra. Bioinfolet 9(3): $360-367$.

Panzade D. S., 2006 Evaluation of some neem products and insecticides against $E$. vitella (Fab.) on okra M.Sc. Agri. Thesis submitted Dr. P.D.K.V. Akola.

Pathak P. H. and S. S. Krishna. 1986. Reproductive efficiency in Earias fabia stoll (Lepidoptera: Noctuidae) affected by neem oil vapour. Apl. Ent. Zoo. 21(2) 347-348.

Priya, B. S and H.P. Misra, 2007. Biopesticides for the management of okra fruit borer, Earias vittella $(\mathrm{Fab})$ pest management in horticultural ecosystem, vol. 13 (2): pp 176-179.

Puranik, T.R., Hadapad, A.B., Salunkhe, G.N., D.S. Pokharakar, 2002. Management of shoot and fruit borer, Leucinodes orbonalis Guenee through Bacillus thuringiensis formulations on brinjal. J. Entomol. Res. 26(3): 229-232.

Reddy, E. and N. Srinivasa 2001. Efficacy of botanicals against brinjal shoot and fruit borer Leucinodes orbonalis Guen. Proceedings of National Symposium on Integrated Pest Management (IPM) in Horticultural crops: New Molecules Biopesticides and Environment, Bangalore 17-19, October, PP. 11-13

Rosaiah, R., 2001. Performance of different botanicals against the pests complex in bhendi. Pestology, 25: 17-19.

Rosaiah, R., 2001a. Evaluation of different botanicals against the pest complex of brinjal. Pestology, 25: 14-16.

Sarode S. V. and A. T. Gabhane. 1994. Performance of neem seed kernal extract with reduced insecticidal dosages on the infestation of okra fruit borer, E. vitella (Fab.) J. Ent. Res. 18(4):327-330

Tomar R.K.S. 1998. Efficacy and Economics of biopesticides and insecticide combination against okra shoot and fruit borer. Indian J. Ent. 60(1): 25-28.

\section{How to cite this article:}

Lakhamapure, A.R., Sh.N.V. Lavhe, P.R. Panchabhai, P.S. Barde and Deotale, R.O. 2018. Assessment of Fruit Infestation of Abelmoschus esculentus by Shoot and Fruit Borer on Weight Basis. Int.J.Curr.Microbiol.App.Sci. 7(12): 3503-3512. doi: https://doi.org/10.20546/ijcmas.2018.712.396 